\title{
Chronic recurrent multifocal osteomyelitis beginning with a solitary lesion of the ilium
}

Ping Zhang ${ }^{1 \dagger}$, Xiao Ying Jia ${ }^{2 \dagger}$, Yun Zhang ${ }^{3}$, John Morelli ${ }^{4}$ and Ze Kun Zhang ${ }^{1 *}$

\begin{abstract}
Background: Chronic recurrent multifocal osteomyelitis (CRMO) is an idiopathic inflammatory disease. The initial lesions are typically found in the metaphyses, generally without periosteal reaction.

Case presentation: We present a case of a 14-year-old female teenager with relapsing and remitting right iliac pain. There was no evidence of infectious organisms, neoplastic processes, or hematologic malignancy based on laboratory tests. Initial computed tomography (CT) and magnetic resonance imaging (MRI) demonstrated atypical periosteal proliferation in the right ilium. Histopathology demonstrated only non-specific chronic inflammation compatible with CRMO. Two years later, this patient developed left humeral pain. MRI and CT images revealed thickening and marrow edema involving the humeral cortex.
\end{abstract}

Conclusions: This case highlights that CRMO can begin as a unifocal lesion and also possibly within the ilium, despite usually being multifocal and involving the long bone metaphysis.

Keywords: Chronic recurrent multifocal osteomyelitis, llium, Periosteal proliferation

\section{Background}

Chronic recurrent multifocal osteomyelitis (CRMO) is an idiopathic inflammatory disease, also known as chronic nonbacterial osteomyelitis. It was first described in 1972 by Giedion et al., in 4 cases of patients suffering from "symmetrical" bone pain [1]. However, the clinical presentation of CRMO has greater diversity. It is best characterized as a skeletal disorder resulting in episodic bone pain of unknown cause. The prevalence of CRMO is estimated as less than 1 in 1,000,000. It mainly affects children and adolescents, especially females (up to 85\%). The lesions are mainly located in the metaphyses and epiphyses of the long bones [2-6]. Some patients might have extra-articular manifestations such as palmoplantar pustulosis, psoriasis, Crohn's disease and other entities $[7,8]$. Radiography is the primary examination in pediatric patients presenting with a limp or pain possibly originating in bone. The initial lesions of CRMO are usually found in the metaphyses, near the growth plates,

\footnotetext{
*Correspondence: zhangzkjia@163.com

${ }^{\dagger}$ Equal contributors

'Department of Radiology, The third Hospital of Hebei Medical University, Hebei Province Biomechanical Key Laborary of Orthopedics, No.139, Ziqiang Road, Shijiazhuang City, Hebei Province 050051, China

Full list of author information is available at the end of the article
}

generally without periosteal reaction. If the lesions have breached the cortices with or without periosteal reaction, they may be misdiagnosed as a tumor, especially the unifocal lesions [9-11].

Here, we describe the case of a 14-year-old girl who initially presented with episodic osseous right ilium bone pain, eventually diagnosed with CRMO after extensive evaluation. The diagnosis in this case was difficult to make because of the unusual initial site and atypical presentation of periosteal proliferation and soft tissue swelling. Knowledge of this unusual case may help skeletal radiologists to consider CRMO as one of the differential diagnoses in patients with similar presentations.

\section{Case presentation}

In July 2013, a 14-year-old girl without history of trauma or medication use presented with a one-yearhistory of right hip pain resulting in limitations of daily activities. The pain was relapsing and remitting in nature, without any associated numbness, weakness, or bowel dysfunction. There was no history of fever, no other musculoskeletal complaints, and no family history of skeletal problems.

On physical examination, the patient experienced obvious pain in the right iliac wing on palpation and had a 
positive Patrick's test (A test performed by having the tested leg flexed and the thigh abducted and externally rotated. If pain is elicited, it is suggestive of a hip joint or the sacroiliac joint disorder). The right hip had a range of motion of approximately 90 degrees of flexion, 10 degrees of abduction, 20 degrees of internal rotation and 30 degrees of external rotation. The skin temperature was normal. Laboratory results revealed a normal white blood cell count, mildly elevated Creactive protein (CRP) of $16.57 \mathrm{mg} / \mathrm{L}$ (normal value, $\leq 10 \mathrm{mg} / \mathrm{L}$ ), and normal erythrocyte sedimentation rate (ESR) of $12.65 \mathrm{~mm} / \mathrm{h}$ (normal value, $0-20 \mathrm{~mm} / \mathrm{h}$ ).

\section{Imaging findings}

Initial radiography demonstrated a mixed lytic and sclerotic lesion of the right ilium with extensive periosteal reaction, suggesting an aggressive process such as a neoplasm (Fig. 1). The MRI revealed that the right ilium was expanded with diffuse bone marrow edema and adjacent soft tissue edema within the iliacus and gluteus minimus muscles (Fig. 2). Findings were concerning for an infiltrative or malignant process, such as lymphoma, Ewing's sarcoma or osteomyelitis. A CT scan demonstrated a markedly atypical periosteal proliferation involving the right ilium with a multifocal lytic lesion (Fig. 2). An open biopsy was subsequently performed followed by surgical bone curettage of right ilium. Histopathology showed a nonspecific fibro-inflammatory infiltrate composed of scattered chronic inflammatory cells including lymphocyte and plasma cells (Fig. 3). There was no evidence of neoplasm. Antibiotic therapy was

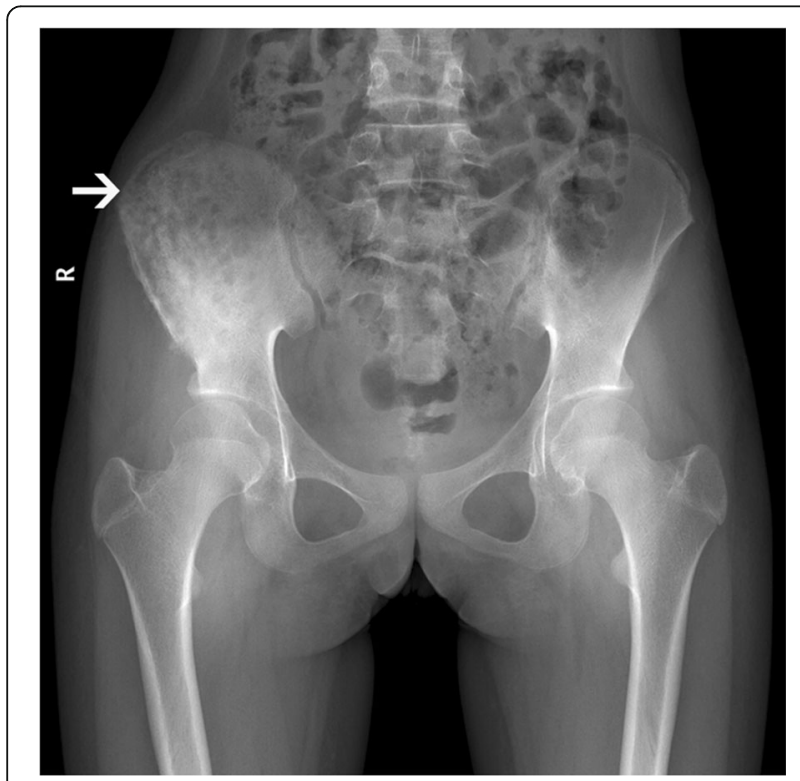

Fig. 1 Anteroposterior radiograph of pelvis shows a predominantly Iytic process with sclerosis and periosteal proliferation in the right iliac wing (white arrow) started with intravenous ceftriaxone and clindamycin hydrochloride. After one week of antibiotic therapy, the patent's clinical signs and symptoms resolved.

\section{Subsequent imaging, pathology, and laboratory findings} In July 2015, the patient was readmitted due to left upper arm pain and a mild fever. On physical examination, there was localized tenderness to the left arm. Full blood count was again normal. The CRP was $58.65 \mathrm{mg} /$ $\mathrm{L}$ (normal value, $\leq 10 \mathrm{mg} / \mathrm{L}$ ), and the ESR was $110 \mathrm{~mm} / \mathrm{h}$ (normal value, $0-20 \mathrm{~mm} / \mathrm{h}$ ). Blood cultures were negative. The upper extremity MRI revealed a left humeral lesion with diffuse low T1 and intermediate to hyper intense T2 signal. There was no evidence of abscess formation in the surrounding soft tissue structures. CT revealed obvious cortical thickening of the left humerus (Fig. 4). A whole-body Technetium-99 m hydroxymethyl disphosphonate (Tc-99mHDP) bone scan was performed to investigate the possibility of multifocal lesions. It showed mild increased activity in the left humerus and obviously increased activity in the right ilium (Fig. 4). The bone biopsy of the left humerus revealed a nonspecific fibro-inflammatory infiltrate and normal hematopoietic marrow without organism growth or evidence of neoplasm. Acid fast and $\mathrm{KoH}$ stains were negative. The flow cytometric analysis showed no evidence of a hematologic malignancy.

\section{Clinical diagnosis}

The biopsies of the lesions were notably negative for malignancy or infection. Based on the progressive nature of the patient's clinical symptoms and imaging findings, she was diagnosed with CRMO. The treatment of CRMO generally involves anti-inflammatory agents targeting symptomatic relief, particularly non-steroidal antiinflammatory medication (i.e. ibuprofen). The patient experienced relief with anti-inflammatory therapy and with local treatment of the right iliac lesion, leading support to the diagnosis.

\section{Discussion and Conclusions}

CRMO is a rare inflammatory bone disease, accounting for $2 \%$ to $5 \%$ of all cases of osteomyelitis [12]. Currently the etiology of CRMO is unknown. Several studies are in progress to investigate its pathogenesis. The generally supported hypothesis suggests a noninfectious inflammatory process as a possible etiology due to the fact that pathogens cannot be found in blood cultures and antibiotics cannot relieve symptoms or alter the disease course $[13,14]$. Autoimmune causes have also been hypothesized as a possible etiology. According to reports, CRMO is associated with peripheral arthritis [15], inflammatory bowel disease [2], and sacroiliitis [8]. It might also represent a special type of juvenile seronegative 

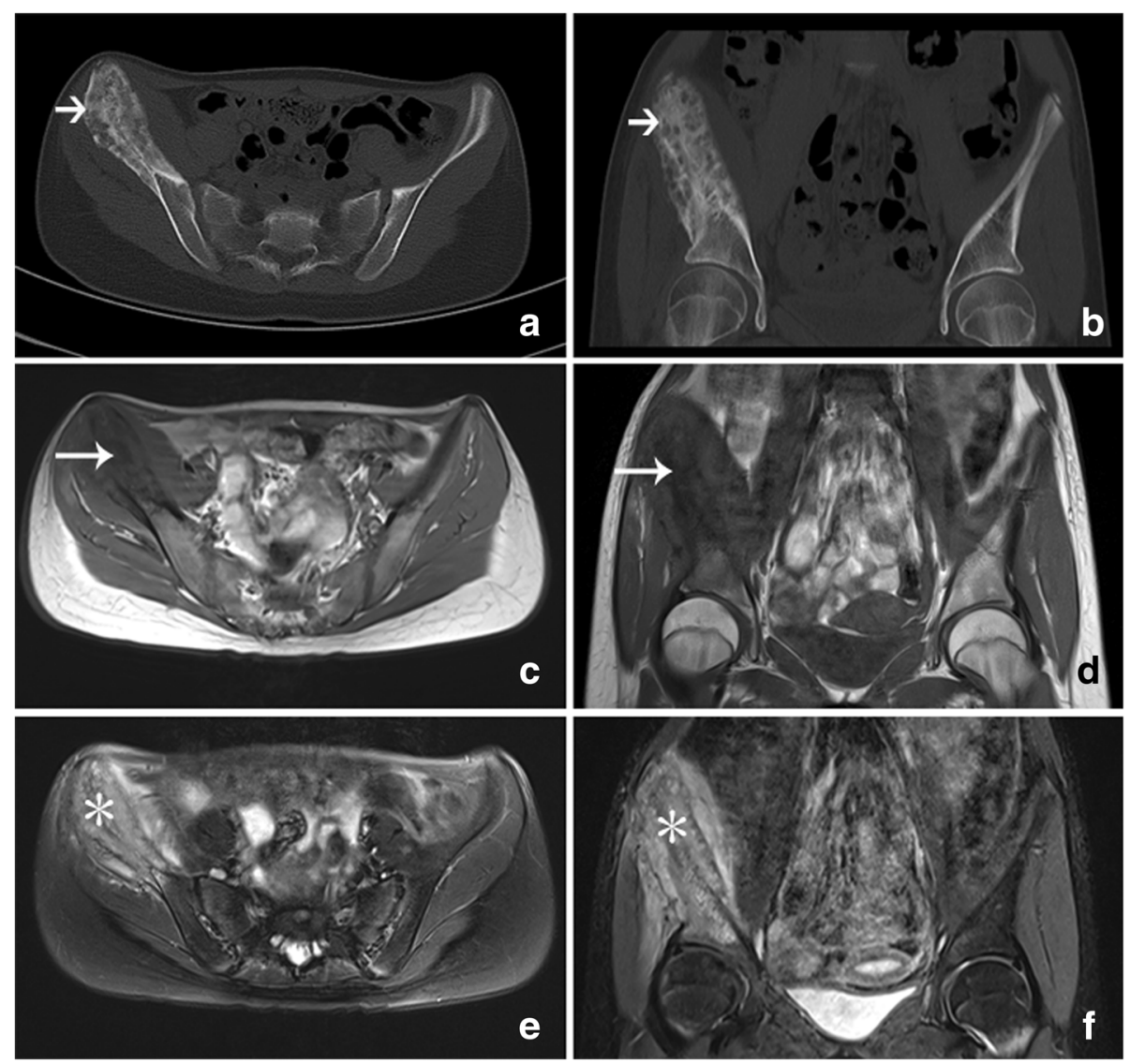

Fig. 2 Axial (a) and reformatted coronal (b) CT images obtained in bone windows illustrate an osteolytic, expanded lesion with mild sclerosis and periosteal proliferation (short arrow). MRI of this lesion presented hypo-signal intensity (long arrow) on T1-weighted images (c, $\mathbf{d}$ ) and scattered hyper-signal intensity (star) on fat suppressed T2-weighted images $(\mathbf{e}, \mathbf{f})$, with soft tissue edema

spondyloarthropathy, as it exhibits some features of SAPHO (synovitis, acne, pustulosis, hyperostosis, and osteitis) syndrome [14].

CRMO is primarily a diagnosis of exclusion based on the clinical symptoms, laboratory tests, imaging and pathological findings. In general, the occurrence of multifocal bone pain is suggestive of CRMO, however

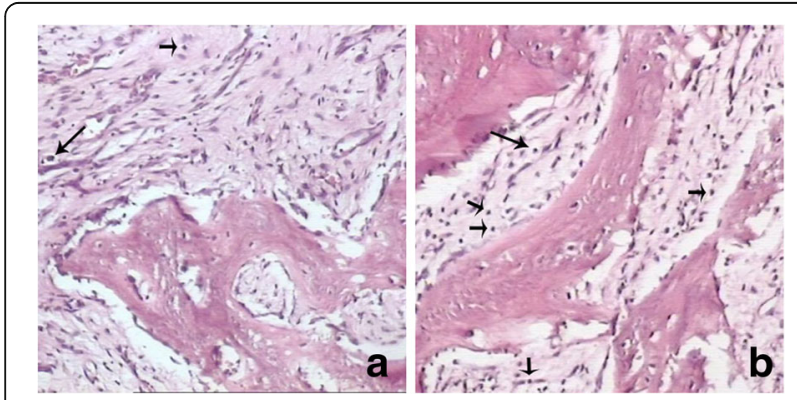

Fig. 3 Microscopic examination of the right ilium biopsy (HE $10 \times 10$ ) shows a non-specific fibro-inflammatory infiltrate composed of scattered chronic inflammatory cells including lymphocyte neutrophils (short arrow $\mathbf{a}, \mathbf{b}$ ) and plasma cells (long arrow $\mathbf{a}, \mathbf{b}$ ) investigations must be performed to rule out other major differential considerations, such as infectious osteomyelitis and bone tumors [9]. The lesions are typically located in the metaphyses of tubular bones, followed by the clavicle and spine $[6,16,17]$. It is unusual for CRMO to begin in a flat pelvic bone such as the ilium; although an initial presentation in the vertebra has been previously reported in children and adults $[18,19]$. It is reported that a CRMO patient with an initial unifocal lesion has a more significant diagnostic delay than a patient with multifocal lesions [4]. The unusual location of the initial solitary lesion (right ilium) in this case added to difficulties in the diagnosis of CRMO. A prolonged and fluctuating course with recurrent episodes of pain is a feature of CRMO. The activity of CRMO may persist for years or even decades. In this case, the left humeral lesion, also contributing to the diagnostic delay.

In general, the initial imaging manifestation of CRMO is lytic destruction in the metaphyses of tubular bones, demarcated by a sclerotic rim. Bone destruction without periosteal reaction or sequestration formation is typical [20-24]. The initial presentation in this case with 


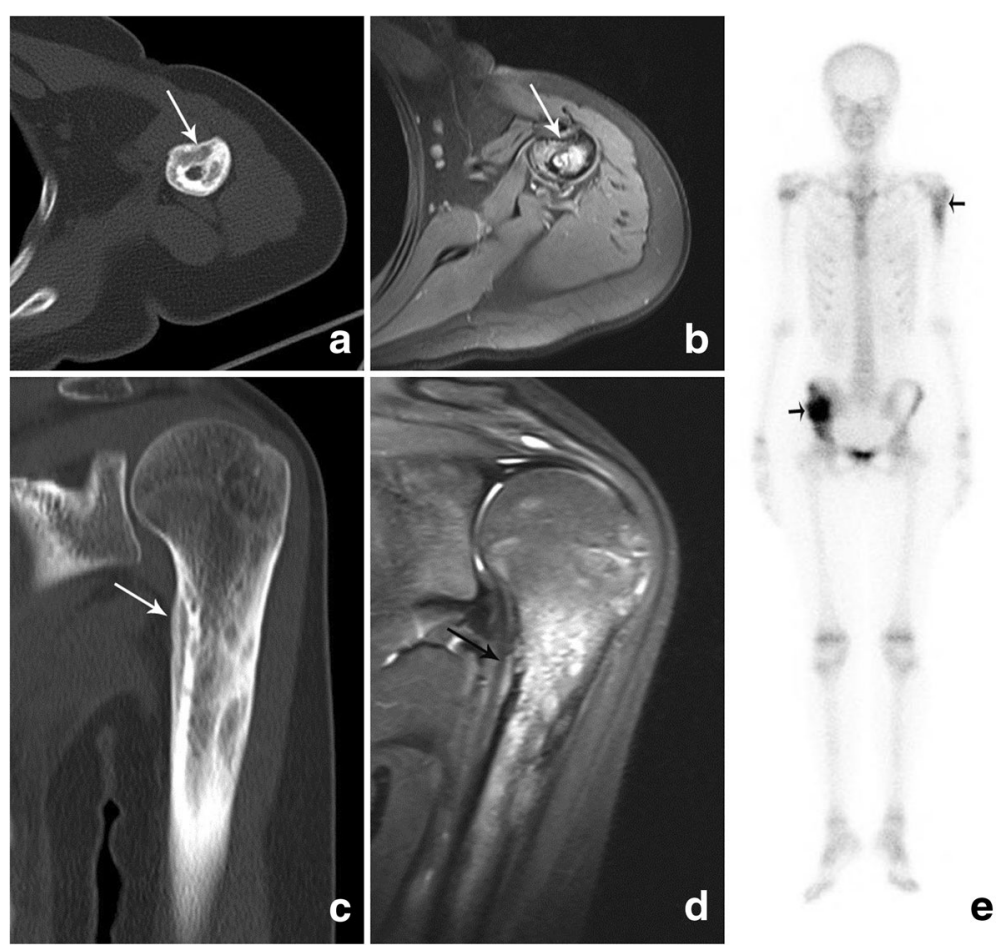

Fig. 4 CT images of the left humerus $(\mathbf{a}, \mathbf{c})$ obtained with bone window and fat-suppressed T2-weighted MR images (b, d) illustrate an osteolytic lesion with periosteal proliferation (white arrow) similar to the initial lesion. Techenetium-99 m methylene diphosphonate bone scintigraphy (e), anterior view of the whole body presents increased radiotracer activity within the right ilium and left humerus (black arrow)

significant periosteal proliferation made it difficult to rule out a malignant tumor, especially Ewing's sarcoma and Langerhans' cell histiocytosis. In CRMO involving tubular bones, abscess formation and soft tissue inflammatory changes are generally absent, whereas in this case there was mild soft tissue inflammation, making it difficult to rule out infectious osteomyelitis. MRI is sensitive to the bone marrow edema and soft tissue inflammatory changes seen in CRMO, but these findings are nonspecific.

Pathologic investigation plays a major role in ruling out other diagnoses, especially when oncologic bone lesions and osteomyelitis cannot be excluded by imaging. Histological investigations show inflammatory changes with granulocytic, lymphocyte, and plasma cell infiltration $[25,26]$. It is impossible to cultivate any infectious organism from CRMO, so antibiotic treatment is useless. Reduction of bone pain with anti-inflammatory therapy does not correspond with radiological remission [27]. It is reported that bisphosphonate therapy has a positive effect on CRMO $[28,29]$. Unfortunately, this patient did not receive bisphosphonate therapy.

\section{Conclusions}

CRMO has no specific clinical, imaging, or pathological findings. The diagnosis depends on a multidisciplinary effort. It is therefore important for skeletal radiologists to be familiar with the variable presentations of CRMO. This case demonstrates that CRMO can present as a solitary lesion of the ilium with significant periosteal proliferation and soft tissue changes. Knowledge of this unusual case may help skeletal radiologists to consider CRMO in their differential diagnosis.

\section{Abbreviations}

CRMO: Chronic recurrent multifocal osteomyelitis; $\mathrm{CT}$ : Computed

tomography; MRI: Magnetic resonance imaging; CRP: C-reactive protein; ESR: Erythrocyte sedimentation rate; Tc-99mHDP: Technetium-99 m hydroxymethyl disphosphonates; SAPHO: Synovitis, acne, pustulosis, hyperostosis, and osteitis

\section{Acknowledgements}

We thank for the patient who agree us to use her image and clinical data.

Funding

None.

\section{Availability of data and materials}

This is a case report of a single patient. To protect privacy and respect confidentially, no raw data have been made available in any public repository. The original operation reports, imaging studies, and outpatient clinic record are retained as per normal procedure within the medical records of our institution.

\section{Authors' contributions}

The manuscript preparation was finished by PZ and XYJ. The manuscript was edited by ZKZ, YZ and JM. All authors have read and approved of the final manuscript.

Competing interests

The authors declare that there are no competing interests. 


\section{Consent for publication}

We conform that the parent of this patient has given his written consent for the case report to be published.

\section{Ethics approval and consent to participate}

The parent of the patient consented to participate this case report.

\section{Author details}

'Department of Radiology, The third Hospital of Hebei Medical University, Hebei Province Biomechanical Key Laborary of Orthopedics, No.139, Ziqiang Road, Shijiazhuang City, Hebei Province 050051, China. ²Department of Emergency, The Hebei general Hospital, Shijiazhuang, Hebei 050051, China. ${ }^{3}$ Department of Outpatient, Chinese people's Armed Police Force 8640 hospital, Dingzhou, Hebei, China. ${ }^{4}$ Department of Radiology, St. Johns Medical Center, Tulsa, OK, USA.

Received: 22 March 2017 Accepted: 1 June 2017

Published online: 06 June 2017

\section{References}

1. Giedion A, Holthusen W, Masel L, et al. Subacute and chronic "symmetrical"osteomyelitis. Ann Radiol. 1972;3:329-42. [Article in Multiple languages]

2. Huber AM, Lam PY, Duffy CM, et al. Chronic recurrent multifocal osteomyelitis: Clinical outcomes after more than five years of follow-up. J Pediatr. 2002:2:198-203.

3. Catalano-Pons C, Comte A, Wipff J, et al. Clinical outcome in children with chronic recurrent multifocal osteomyelitis. Rheumatology. 2008:9:1397-9.

4. Kaiser D, Bolt I, Hofer M, et al. Chronic nonbacterial osteomyelitis in children: a retrospective multicenter study. Pediatr Rheumatol. 2015;1:1-7.

5. Schultz C, Holterhus PM, Seidel A, et al. Chronic recurrent multifocal osteomyelitis in children. Pediatr Infect Dis. 1999;11:1008.

6. Costa-Reis P, Sullivan KE. Chronic recurrent multifocal osteomyelitis. J Clin Immunol. 2013;33:1043-56

7. Jansson A, Renner ED, Ramser J, et al. Classification of non-bacterial osteitis: retrospective study of clinical, immunological and genetic aspects in 89 patients. Rheumatology. 2007:1:154-60.

8. Vittecoq O, Said LA, Michot C, et al. Evolution of chronic recurrent multifocal osteitis toward spondylarthropathy over the long term. Arthritis Rheum. 2000;1:109-19.

9. Wipff J, Adamsbaum C, Kahan A, et al. Chronic recurrent multifocal osteomyelitis. Joint Bone Spine. 2011;78:555-60.

10. Gikas PD, Islam L, Aston W, et al. Nonbacterial osteitis: a clinical histopathological, and imaging study with a proposal for protocol-based management of patients with this diagnosis. J Orthop Sci. 2009;5:505-16.

11. Coinde E, David L, Cottalorda J, et al. Chronic recurrent multifocal osteomyelitis in children: a report of 17 cases. Arch Pediatr. 2001;6:577-83.

12. Girschick HJ, Krauspe R, Tschammler A, et al. Chronic recurrent osteomyelitis with clavicular involvement in children: diagnostic value of different imaging techniques and therapy with non-steroidal anti-inflammatory drugs. Eur JPediatr. 1998;1:28-33.

13. Cyrlak D, Pais MJ. Chronic recurrent multifocal osteomyelitis. Skeletal Radiol. 1986:1:32-9.

14. Jurik AG. Chronic Recurrent Multifocal Osteomyelitis. Semin Musculoskelet Radiol. 2004:3:243-53.

15. Beretta-Piccoli BC, Sauvain MJ, Gal I, et al. Synovitis, acne, pustulosis, hyperostosis, osteitis (SAPHO) syndrome in childhood: a report of ten cases and review of the literature. Eur J Pediatr. 2000;8:594-601.

16. Roderick MR, Ramanan AV. Chronic recurrent multifocal osteomyelitis. Adv Exp Med Biol. 2013;764:99-107.

17. Nemcova D, Koskova E, Macku M, et al. Chronic recurrent multifocal osteomyelitis (CRMO): clinical features and outcome in 21 Czech and Slovak children. Clin Exp Rheumatol. 2011;2:391.

18. Hong CW, Hsiao EC, Horvai AE, et al. Chronic recurrent multifocal osteomyelitis with an atypical presentation in an adult man. Skelet Radiol. 2015:44:1359-64.

19. Anderson SE, Heini P, Sauvain MJ, et al. Imaging of chronic recurrent multifocal osteomyelitis of childhood first presenting with isolated primary spinal involvement. Skelet Radiol. 2003;6:328-36.

20. Jurik AG, Egund N. MRI in chronic recurrent multifocal osteomyelitis. Skelet Radiol. 1997:4:230-8
21. Probst FP, Bj rksten B, Gustavson KH. Radiological aspect of chronic recurrent multifocal osteomyelitis. Ann Radiol. 1978:21:115-25.

22. Gustavson KH, Wilbrand HF. Chronic symmetric osteomyelitis. Report of a case. Acta Radiol Diagn (Stockh). 1974;5:551-7.

23. Meyer zu Reckendorf G, Milon E, Pous JG. Chronic Recurrent Multifocal Osteomyelitis (C.R.M.O.). A Case Report and Review of Literature. Eur J Pediatr Surg. 1996;5:312-5.

24. Sabbioni G, Piccolo ND, Gualdrini G. Chronic recurrent multifocal and aspecific osteomyelitis: a case report. Musculoskeletal Surg. 2010;1:45-7.

25. Sadeghi E. Chronic Recurrent Multifocal Osteomyelitis: A Case Report. Iran Red Crescent Med J. 2011;1:47-51.

26. Bjorksten B, Boquist L. Histopathological aspects of chronic recurrent multifocal osteomyelitis. J Bone Joint Surg Br. 1980;62(3):376-80.

27. Beck C, Morbach H, Beer M, et al. Chronic nonbacterial osteomyelitis in childhood: prospective follow-up during the first year of anti-inflammatory treatment. Arthritis Res Ther. 2010;2:1-11.

28. Compeyrot-Lacassagne S, Rosenberg AM, Babyn P, et al. Pamidronate treatment of chronic noninfectious inflammatory lesions of the mandible in children. J Rheumatol. 2007;7:1585-9.

29. Simm PJ, Allen RC, Zacharin MR. Bisphosphonate Treatment in Chronic Recurrent Multifocal Osteomyelitis. J Pediatr. 2008:4:571-5.

\section{Submit your next manuscript to BioMed Central and we will help you at every step:}

- We accept pre-submission inquiries

- Our selector tool helps you to find the most relevant journal

- We provide round the clock customer support

- Convenient online submission

- Thorough peer review

- Inclusion in PubMed and all major indexing services

- Maximum visibility for your research

Submit your manuscript at www.biomedcentral.com/submit 